\title{
ALFALFA H $\alpha$ Reveals How Galaxies Use Their Hi Fuel
}

\section{Anne Jaskot, ${ }^{1}$ Sally Oey, ${ }^{2}$ John Salzer, ${ }^{3}$ Angie Van Sistine,${ }^{3}$ Eric Bell, ${ }^{2}$ and Martha Haynes ${ }^{4}$}

\author{
${ }^{1}$ Dept. of Astronomy, Smith College, Northampton, MA 01063, USA \\ email: ajaskot@smith.edu \\ ${ }^{2}$ Dept. of Astronomy, University of Michigan, Ann Arbor, MI 48109, USA \\ ${ }^{3}$ Dept. of Astronomy, Indiana University, Bloomington, IN 47405, USA \\ ${ }^{4}$ Center for Astrophysics and Planetary Science, Cornell University, Ithaca, NY 14853, USA
}

\begin{abstract}
Atomic hydrogen traces the raw material from which molecular clouds and stars form. With 565 galaxies from the ALFALFA $\mathrm{H} \alpha$ survey, a statistically complete subset of the ALFALFA survey, we examine the processes that affect galaxies' abilities to access and consume their Hi gas. On galaxy-wide scales, Hi gas fractions correlate only weakly with instantaneous specific star formation rates (sSFRs) but tightly with galaxy color. We show that a connection between dust and Hi content, arising from the fundamental mass-metallicity-Hi relation, leads to this tight color correlation. We find that disk galaxies follow a relation between stellar surface density and Hi depletion time, consistent with a scenario in which higher mid-plane pressure leads to more efficient molecular cloud formation from HI. In contrast, spheroids show no such trend. Starbursts, identified by $\mathrm{H} \alpha$ equivalent width, do not show enhanced HI gas fractions relative to similar mass non-starburst galaxies. The starbursts' shorter Hi depletion times indicate more efficient consumption of HI, and galaxy interactions drive this enhanced star formation efficiency in several starbursts. Interestingly, the most disturbed starbursts show greater enhancements in Hi gas fraction, which may indicate an excess of HI at early merger stages. At low galaxy stellar masses, the triggering mechanism for starbursts is less clear; the high scatter in efficiency and sSFR among low-mass galaxies may result from periodic bursts. We find no evidence for depleted Hi reservoirs in starbursts, which suggests that galaxies may maintain sufficient Hi to fuel multiple starburst episodes.
\end{abstract}

Keywords. galaxies: evolution, galaxies: general, galaxies: interactions, galaxies: ISM, galaxies: starburst, radio lines: galaxies, surveys

The ALFALFA $\mathrm{H} \alpha$ survey (Van Sistine et al.2015) probes the Hi content, star formation rates (SFRs), and $\mathrm{H} \alpha$ and $R$-band morphologies of a statistically complete subset of HI-detected galaxies from the ALFALFA survey (Giovanelli et al.2005). Here, we present results from the 565 "Fall-Sky" ALFALFA H $\alpha$ galaxies (Jaskot et al.2015).

Starburst galaxies, identified by $\mathrm{H} \alpha$ equivalent width, have Hi gas fractions comparable to similar mass non-starbursts and show no signs of Hi depletion. Galaxy interactions may lead to efficient $\mathrm{HI}-\mathrm{to}-\mathrm{H}_{2}$ conversion in several of the starbursts. Only two starbursts have $M_{H I} / M_{*}$ more than $2 \sigma$ above the mean value for their stellar mass, and these starbursts also exhibit the strongest morphological disturbances. During an interaction, inflowing Hi may initially raise galaxies' Hi gas fractions. After coalescence, as morphological disturbances weaken, $\mathrm{H}_{2}$ conversion may eliminate any Hi excess.

For the full "Fall-Sky" sample, $M_{H I} / M_{*}$ correlates weakly with specific SFR (sSFR). In contrast, the strong correlation of color with $M_{H I} / M_{*}$ (Fig. 1) implies a link between HI and recent star formation (e.g., Catinella et al.2010; Huang et al.2012). However, after correcting for dust (Fig. 1), the HI-color trend noticeably weakens. The mass-metallicity-Hi relation (Bothwell et al.2013) may explain the HI-color trend. High-mass galaxies tend to have high metallicities (and high dust reddening), low sSFRs, and low $M_{H I} / M_{*}$.

Disk galaxies with higher stellar surface densities $\left(\Sigma_{*}\right)$ have shorter HI depletion times 

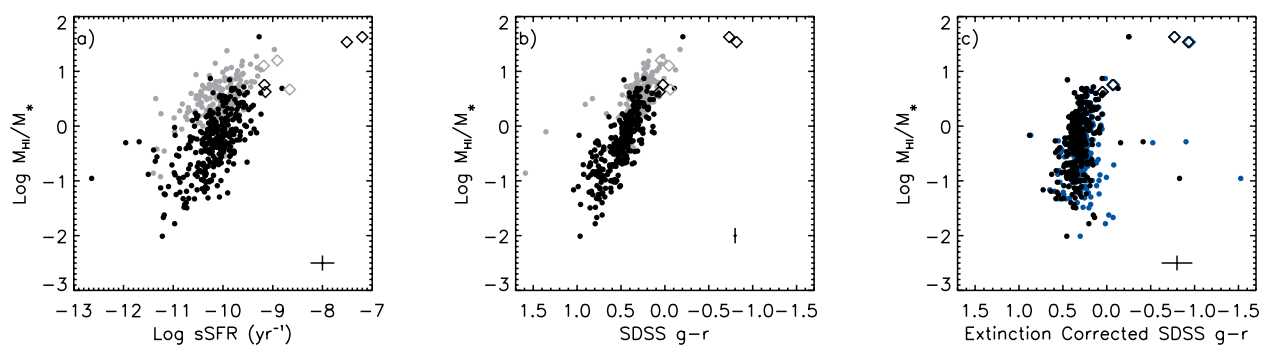

Figure 1. (a) $M_{H I} / M_{*}$ vs. sSFR with WISE $12 \mu \mathrm{m}$ detections in black and non-detections in gray. Diamonds indicate starburst galaxies. (b) $M_{H I} / M_{*}$ vs. SDSS $g-r$. (c) $M_{H I} / M_{*}$ vs. SDSS $g-r$ color, after correcting for extinction using the WISE $12 \mu$ m luminosities (Wen et al.2014) and extinction laws from Calzetti (2000; black) and Cardelli et al.(1989; blue).

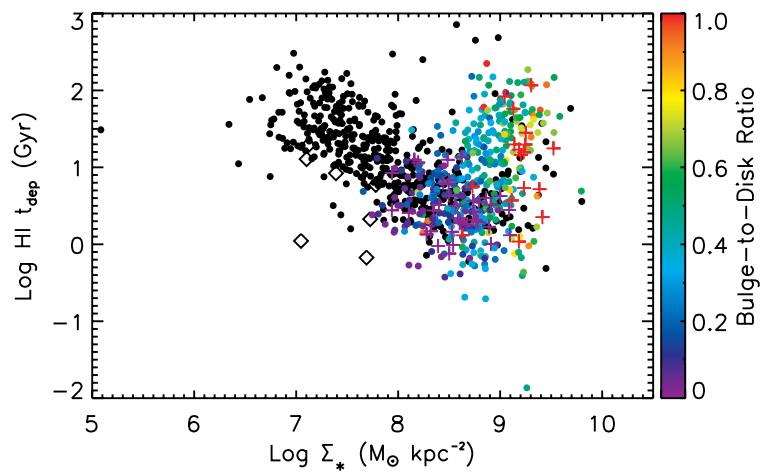

Figure 2. Hi depletion time vs. $\Sigma_{*}$ for the ALFALFA $\mathrm{H} \alpha$ non-starbursts (black circles) and starbursts (diamonds) and the higher mass GASS sample (Catinella et al.2010; colored circles and crosses). The GASS galaxies are colored from the Simard et al.(2011) SDSS fits by bulge-to-disk ratio (circles), Sérsic index $\geqslant 2$ (red crosses), and Sérsic index $<2$ (purple crosses).

( $M_{H I} /$ SFR; Fig. 2), but spheroids do not follow this trend. In disks, higher mid-plane pressure at higher $\Sigma_{*}$ may promote molecular cloud formation from Hi (Blitz \& Rosolowsky 2006). Low-mass galaxies show more scatter in both Hi depletion time and sSFR, which may indicate cyclical bursts of star formation.

\section{References}

Blitz, L., \& Rosolowsky, E. 2006, ApJ, 650, 933

Bothwell, M.S., Maiolino, R., Kennicutt, R., et al. 2013, MNRAS, 433, 1425

Calzetti, D., Armus, L., Bohlin, R.C., et al. 2000, ApJ, 533, 682

Cardelli, J.A., Clayton, G.C, \& Mathis, J. S. 1989, ApJ, 345, 245

Catinella, B., Schiminovich, D., Kauffmann, G., et al. 2010, MNRAS, 403, 683

Giovanelli, R., Haynes, M.P., Kent, B.R., et al. 2005, AJ, 130, 2598

Huang, S., Haynes, M.P., Giovanelli, R., \& Brinchmann, J. 2012, ApJ, 756, 113

Jaskot, A.E., Oey, M.S., Salzer, J.J., et al. 2015, ApJ, 808, 66

Simard, L., Mendel, J.T., Patton, D.R., et al. 2011, ApJS, 196, 11

Van Sistine, A., Salzer, J.J., Sugden, A., et al. 2015, ApJ, Submitted

Wen, X.-Q., Wu, H., Zhu, Y.-N., et al. 2014, MNRAS, 438, 97 